\title{
The Inhibitory Effects of PSS-Loaded Nanoparticles on the Dysfunction of Cardiac Microvascular Endothelia in Rats with Diabetic Cardiomyopathy
}

\author{
AN Nina, YU Luyan, HU Yi, FENG Wenjing, SHI Shujuan, CHEN Honghua*, \\ and MAO Yongjun *
}

Department of Geriatrics, the Affiliated Hospital of Qingdao University, Qingdao 266003, China

(Received September 6, 2018; revised October 22, 2018; accepted October 28, 2018)

(๑) Ocean University of China, Science Press and Springer-Verlag GmbH Germany 2019

\begin{abstract}
Propylene glycol alginate sodium sulfate-loaded nanoparticles (PSS-NP) has been shown potential to prevent the microvascular endothelial injuries caused by diabetic cardiomyopathy (DCM). In this study, we aimed to investigate the effects of PSS-NP on the dysfunction of cardiac microvascular endothelia in streptozotocin (STZ)-induced DCM rat model. Echocardiographic measurements showed a significant improvement of cardiac function in the PSS-NP-treated group. Our results revealed that the abnormalities of cardiac systolic and diastolic functions were suppressed by the treatments of prostaglandin E1 (PGE1), low molecular weight heparin (LMWH), PSS and PSS-NP. Our comparison analysis indicated that PSS-NP showed the strongest inhibitory effects on microvascular endothelial injuries. Transmission electron microscopy analysis demonstrated that PSS-NP protected the cardiac microvascular endothelium and further improved endothelium dysfunction in DCM rats. ELISA and Western blot assays further showed a high efficiency of improving cardiac microvascular endothelial dysfunction with PSS-NP. Our results demonstrated that PSS-NP increased the protein expression of phosphatidylinositol 3-kinase (PI3K)-p85 and vascular endothelial growth factor (VEGF)-A, and the phosphorylation of protein kinase B (Akt) and endothelial nitric oxide synthase (eNOS), which were involved in the amelioration of cardiac microvascular endothelial dysfunction. These data suggest that PSS-NP may be a novel approach to the treatment the coronary microcirculation disorder diseases such as DCM.
\end{abstract}

Key words PSS-loaded nanoparticles; marine drug; DCM; microvascular; endothelial dysfunction

\section{Introduction}

Diabetic cardiomyopathy (DCM) is a deadly disease with complicated causes and high death rate in the patients with diabetes mellitus (DM) (Huynh et al., 2014; Farhangkhoee et al., 2006). Pathologically, cardiac microvascular lesion is one of the characteristic findings of DCM and could lead to myocardial ischemia and finally result in heart failure (HF) (Farhangkhoee et al., 2006; Yoon et al., 2005; Forbes et al., 2013). Endothelium is the key target tissue of microvascular lesion in DCM, and endothelial dysfunction is one of the most crucial pathophysiological bases of microvascular lesion (Kawamura et al., 2017; Zoungas et al., 2010).

The oceans, which cover about 71 percent of the earth's surface area, are a vast reservoir of life. With the deep development of marine resources, the research and development on marine drugs attract more and more attention, especially the bioactive marine polysaccharides.

\footnotetext{
* Corresponding authors. E-mail: qyfychh@163.com

E-mail: mmc168@126.com
}

There are increasing evidences that propylene glycol alginate sodium sulfate (PSS) is a marine sulfated polysaccharide which shows high biological activities of anti-coagulation, anti-oxidative stress and anti-inflammation (Zeng et al., 2016; Li et al., 2013). In 1980s, PSS was proved in China for treatment of hypercoagulable diseases. However, the clinical use of PSS has been gradually reduced because of its low bioavailability and side effects (Li et al., 2013). In order to improve the efficacy of PSS, scientists developed a new preparation of PSS-loaded nanoparticles (PSS-NP). PSS-NP has been found to possess more advantages in bioavailability, stability and targeting (Li et al., 2013; Mizrahy et al., 2012; Feng et al., 2009).

It has been well documented that the phosphatidylinositol 3-kinase/protein kinase B/endothelial nitric oxide synthase/vascular endothelial growth factor A (PI3K/ Akt/eNOS/VEGFA) signaling pathway plays a crucial role in improving the vascular endothelial dysfunction, which can affect cell proliferation, apoptosis and oxidative stress (Simons et al., 2016; Maillet et al., 2013; Wu et al., 2009; Chen et al., 2005; Alp et al., 2003). In this study, we aimed to investigate whether PSS-NP could improve car- 
diac microvascular endothelial function via regulating the $\mathrm{PI} 3 \mathrm{~K} / \mathrm{Akt} / \mathrm{eNOS} / \mathrm{VEGFA}$ signaling pathway in the streptozotocin (STZ)-induced DCM rat model.

\section{Materials and Methods}

\subsection{DCM Rat Model}

All experimental protocols were approved and conducted in accordance with the Ethical Committee of Experimental Animal Care, Marine Biomedical Research Institute of Qingdao. Thirty-six specific pathogen-free (SPF) male Sprague-Dawley (SD) rats (8 weeks, $150-200$ g, Jinan Peng Yue Experimental Animal Breeding Co., Ltd) were used in this study. Six rats were randomly selected as control group. Thirty of the rats were given a single intraperitoneal injection of STZ (Sigma Corporation, USA) at $65 \mathrm{mg} \mathrm{kg}^{-1}$ after fasting overnight to induce DM (Litwin et al., 1990; Dong et al., 2012). Rats in control group were injected with the same volume of citrate buffer. At the 3rd, 10th day and 8th week after STZ administration, the posterior orbital venous plexus fasting blood glucose levels were measured by Glucose Assay Kit (Glucose Oxidase Method) (Shanghai Rongsheng Biotechnology Co., Ltd, China). Eight weeks after STZ administration, the rats with the fasting blood glucose levels exceeding $16.7 \mathrm{mmol} \mathrm{L}^{-1}$ and with cardiac dysfunction (confirmed by echocardiography) were considered DCM rats (Wang et al., 2013; Fang et al., 2004; Liu et al., 2012).

\subsection{Animal Treatments}

After DCM rat model was established, the DCM rats were randomly divided into five groups ( $n=6$ per group): (1) DCM: DCM rats were treated with $2 \mathrm{~mL}$ normal saline; (2) DCM+PGE1: DCM rats were treated with PGE1 (5 $\mu \mathrm{g} \mathrm{kg}^{-1}$ ) (Beijing Tide Pharmaceutical Co., Ltd, China) in $2 \mathrm{~mL}$ normal saline; (3) DCM+LMWH: DCM rats were treated with LMWH (150 $\mathrm{IU} \mathrm{kg}^{-1}$ ) (Alfa Wassermann Pharmaceutical Company, Italy) in $2 \mathrm{ml}$ normal saline; (4) DCM+PSS: DCM rats were treated with PSS $\left(20 \mathrm{mg} \mathrm{kg}^{-1}\right)$ (Qingdao Haier Pharmaceutical Co., Ltd, China) in $2 \mathrm{ml}$ normal saline; and (5) DCM+PSS-NP: DCM rats were treated with PSS-NP $\left(20 \mathrm{mg} \mathrm{kg}^{-1}\right)$ (Ocean University of China, China) in $2 \mathrm{ml}$ normal saline. Control rats were treated with $2 \mathrm{~mL}$ saline (Hasegawa et al., 2015; Green et al., 1994; Xin et al., 2016). All rats were treated with the corresponding medicines or saline via intraperitoneal injection once a day for 4 consecutive weeks.

\subsection{Monitoring of Fasting Blood Glucose and Body Weight}

During the treatment, the fasting blood glucose and the body weight were dynamically monitored on the 2 nd and 4th week, respectively. Serum levels of fasting blood glucose were measured by Glucose Assay Kit according to the protocol information (Glucose Oxidase Method) (Shanghai Rongsheng Biotechnology Co., Ltd, China).

\subsection{Echocardiography Analysis}

To assess the effects of PSS-NP on cardiac function of DCM rats, transthoracic echocardiography was executed by Vevo 2100 ultrasound device (Visual-Sonics, Toronto, Canada) after 4 weeks of treatment as described previously (Wang et al., 2013; Yin et al., 2014; Wu et al., 2017), briefly, rats were anesthetized with isoflurane vaporized in oxygen $(2.5 \%$ induction, $1.0 \%$ maintenance) and the cardiac function images and indexes were obtained under M-mode echocardiography from the short axis view of the level of papillary muscles combined with Pulsed Doppler echocardiography from an apical four-chamber view: Left ventricular internal diameter in diastole (LVIDd), left ventricular internal diameter in systole (LVIDs), fractional shortening (FS), left ventricular ejection fraction (LVEF) and $\mathrm{E}$ wave to $\mathrm{A}$ wave ratio (E/A ratio). All data represent the average of three cardiac cycles.

\subsection{Determination of Serum eNOS, VEGFA and Nitric Oxide (NO) Levels}

After 4 weeks of treatment, blood samples were collected and then centrifuged at $2500 \mathrm{r} \mathrm{min}^{-1}$ for $15 \mathrm{~min}$. Serum was used to assess the concentration of eNOS and VEGFA by ELISA kits (Elabscience Biotechnology Co., Ltd, China). NO concentration was detected by NO assay kit according to the protocol information (Nitrate reductase method) (Jiancheng Bioengineering Institute, Nanjing, China). All procedures were executed according to the manufacturer's instructions.

\subsection{Transmission Electron Microscopy}

After 4 weeks of treatment, transmission electron microscopy was used to determine the ultrastructure of cardiac microvascular endothelium as previously described (Zhang et al., 2017; Stefek et al., 2000). Briefly, the apical tissue of the heart was cut into $1 \mathrm{~mm}^{3}$ cubes and fixed in $2.5 \%$ glutaraldehyde overnight at $4^{\circ} \mathrm{C}$. After a series of procedures including fixation, washing, stepwise alcohol dehydration, embedding, polymerization, sectioning and staining, ultrathin sections were observed under a transmission electron microscopy (JEM-1200EX TEM, Japan).

\subsection{Western Blot Analysis}

Western blot assay was performed as previously described (Hoehn et al., 2008; Saito et al., 2018). Briefly, total protein samples were extracted from left ventricular myocardium and the protein concentration was determined by BCA assay. After samples were homogenized in loading buffer, protein extracts were separated on a $12 \%$ SDS-PAGE and electro-transferred onto NC membranes. After blocking with 5\% skim milk, samples were probed with PI3K-p85, Akt, pS473-Akt, eNOS, p-eNOS (Cell Signaling Technology, USA) and VEGFA antibodies (Abcam, UK) (1:500); equal protein loading was confirmed by reprobing the membranes with an antibody to 
$\beta$-actin (Merck Millipore, Germany) (1:500). The primary antibodies were incubated with secondary horseradish peroxidase-conjugated antibody (Jackson ImmunoResearch, USA) (1:5000). Antigen-antibody complexes were visualized with Immobilon Western Chemiluminescent HRP substrate (Merck Millipore, Germany), and the intensity of the protein bands was quantified with Quantity One software (Bio-Rad, USA).

\subsection{Statistical Analysis}

All data were performed as mean $\pm \mathrm{SD}$ and analyzed using One-Way ANOVA. $P<0.05$ was considered to be

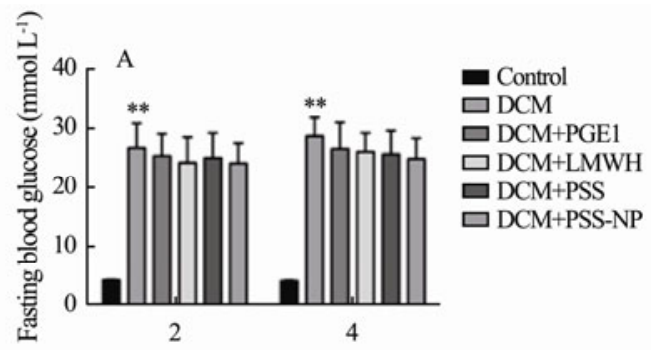

statistically significant. All statistical tests were expressed using GraphPad Prism software (GraphPad Software, San Diego, CA).

\section{Results}

\subsection{The Improvement of Fasting Blood Glucose and Body Weight}

The levels of fasting blood glucose and body weight (Fig.1A and Fig.1B) were measured to access the effects of PSS-NP therapy on glucose metabolism and body weight changes. As expected, DCM group had significantly

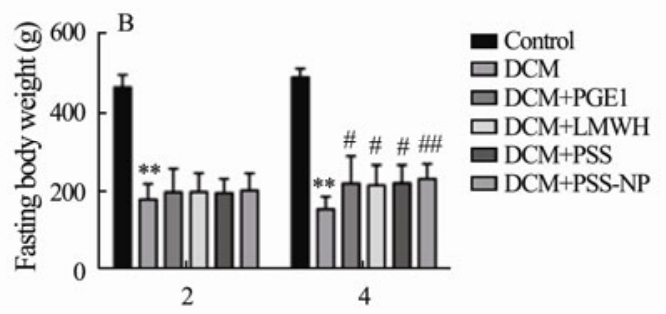

Fig.1 The results of fasting blood glucose and body weight in all groups. (A and B) The fasting blood glucose and the body weight were dynamically monitored on the 2 nd and 4 th week during the treatment. Results are displayed as mean $\pm \mathrm{SD}, \mathrm{n}=6$ per group. ${ }^{* *} P<0.01$ vs Control group, ${ }^{\#} P<0.05$ or ${ }^{\# \#} P<0.01$ vs DCM group.

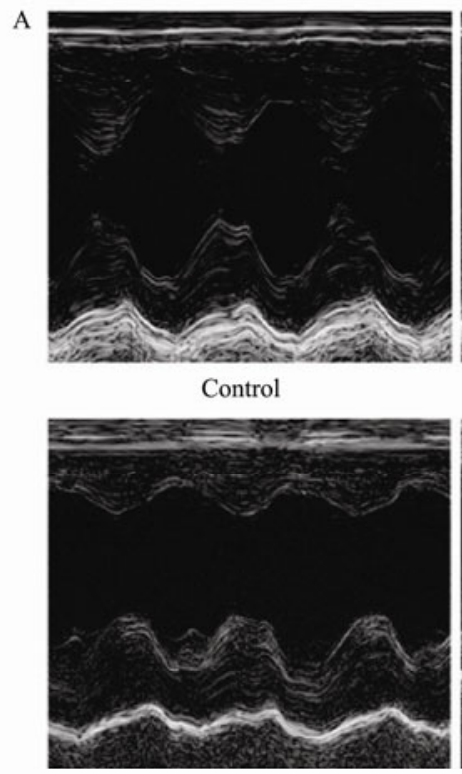

$\mathrm{DCM}+\mathrm{LMWH}$

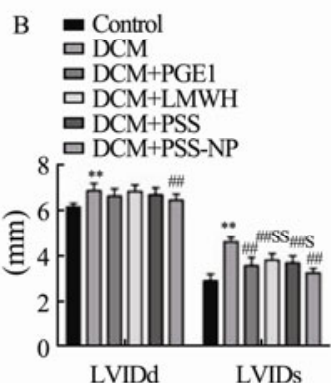

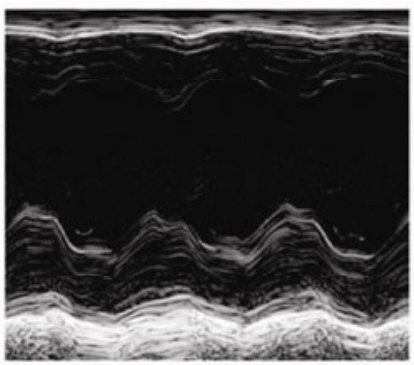

DCM

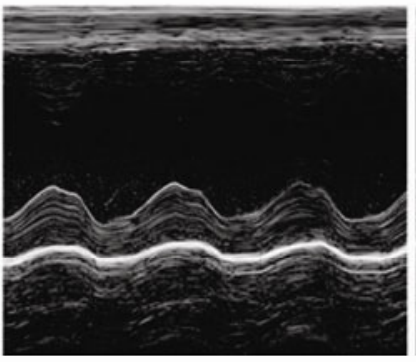

DCM+PSS

C $=$ Control

$\square \mathrm{DCM}$
$\square \mathrm{DCM}+\mathrm{PG}$

口DCM+LMWH

口DCM+PSS

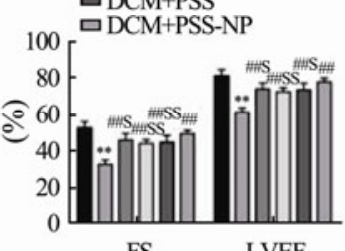

FS

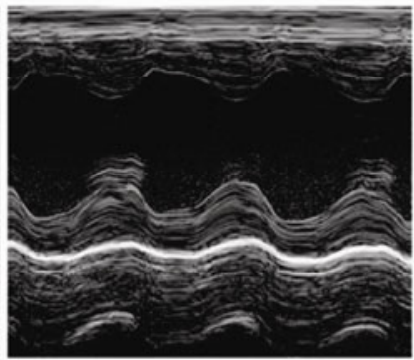

$\mathrm{DCM}+\mathrm{PGE} 1$

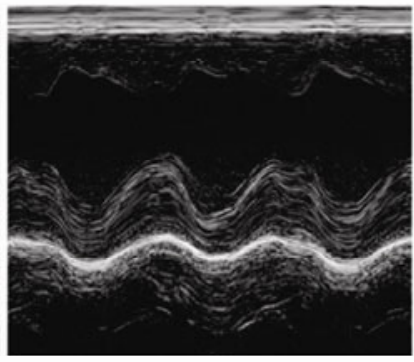

DCM+PSS-NP

D

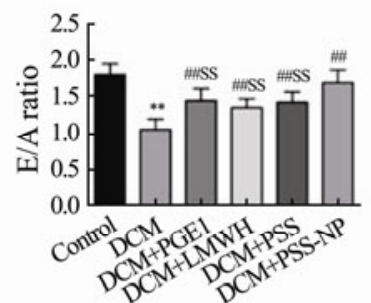

Fig. 2 Changes of cardiac function in all groups. (A) Representative echocardiography images. (B-D) The cardiac systolic and diastolic function indexes: LVIDd, LVIDs, FS, LVEF and E/A ratio. Results are expressed as mean \pm SD, $n=6$ per group. ${ }^{* *} P<0.01 v s$ Control group, ${ }^{\#} P<0.01 v s$ DCM group, ${ }^{\$} P<0.05$ or ${ }^{\$ \$} P<0.01 v s$ DCM+PSS-NP group. 
higher fasting blood glucose levels and lower fasting body weight than those of the control group $(P<0.01)$. However, compared with DCM group, there was no statistical difference in fasting blood glucose in DCM+PGE1 group, $\mathrm{DCM}+\mathrm{LMWH}$ group, DCM+PSS group and DCM+ PSS-NP group $(P>0.05)$. The fasting body weight were obviously increased $(P<0.05)$ in all of the treatment groups, particularly in the DCM+PSS-NP group $(P<0.01)$. Moreover, there were no statistical differences among $\mathrm{DCM}+$ PSS-NP group and other treatment groups in fasting blood glucose and body weight $(P>0.05)$. These results showed that all treatments modified the fasting body weight without altering the fasting blood glucose levels.

\subsection{The Alleviation of Cardiac Dysfunction by PSS-NP}

M-mode images revealed that DCM group had a significant decrease in cardiac wall motion compared with control group (Fig.2A). However, the abnormal cardiac wall motion was alleviated in all of the treatment groups. These results suggest that PSS-NP could improve the cardiac wall motion.

As expected, compared with control group, the DCM group showed markedly higher LVIDd and LVIDs but lower FS, LVEF and E/A ratio $(P<0.01)$ (Figs.2B-2D). All of the treatment groups had significant lower LVIDs and higher FS, LVEF and E/A ratio than those of DCM group $(P<0.01)$. However, it was observed that only the LVIDd in DCM+PSS-NP group showed overt improvement compared with DCM group $(P<0.01)$. All these indexes in the DCM+PSS-NP group were significantly superior to other treatment groups $(P<0.05$ or $P<0.01)$, which indicated that PSS-NP had the best effect on the improvement of cardiac systolic and diastolic function.

\subsection{The Protective Effects of the Cardiac Microvas- cular Endothelium by PSS-NP}

As shown in Fig.3, in control group, the surface of cardiac microvascular was smooth and endothelial cell morphology was completely normal. However, in DCM group, the nuclei of endothelial cells were enlarged with various shapes and the chromatin condensed in margin. Cell membrane showed finger-shaped protuberances to the lumen. All treatments effectively alleviated the injuries. In fact, the alterations of cardiac microvascular endothelium morphology in DCM+PSS-NP group were approximately similar to those of the control group. These findings demonstrated that PSS-NP protected the cardiac microvascular endothelium and further improved microvascular endothelium dysfunction in DCM rats.

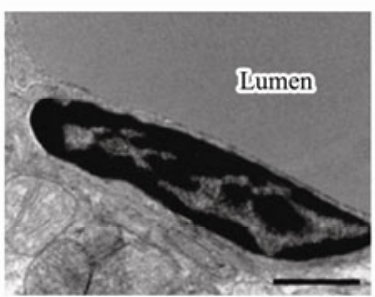

Control

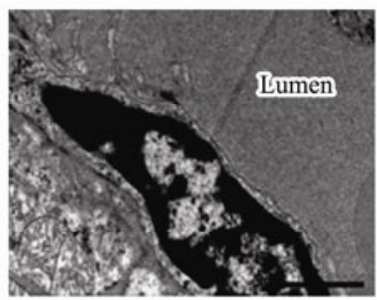

$\mathrm{DCM}+\mathrm{LMWH}$

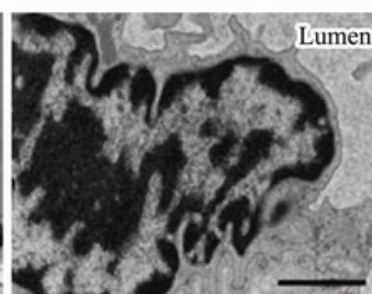

DCM

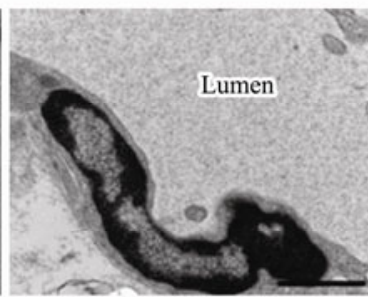

$\mathrm{DCM}+\mathrm{PSS}$

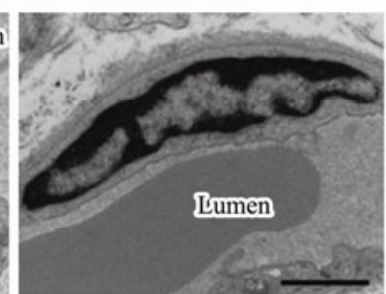

$\mathrm{DCM}+\mathrm{PGE} 1$

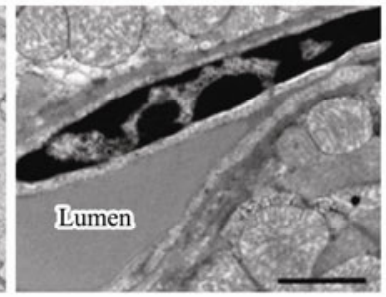

DCM+PSS-NP

Fig.3 Changes of cardiac microvascular endothelial ultrastructure in all groups. Representative electron micrographs (Scale bar $=1 \mu \mathrm{m}$, magnification $20000 \times), \mathrm{n}=6$ per group.

\subsection{The Up-Regulation of eNOS, NO and VEGFA, and the Activation of PI3K/Akt/eNOS/VEGFA Pathway by PSS-NP}

As shown in Figs.4A-4C, the decreased concentrations of eNOS, NO and VEGFA were detected in DCM group compared with control group $(P<0.01)$. All treatment groups had dramatically higher concentrations of eNOS, NO and VEGFA than DCM group $(P<0.05$ or $P<0.01)$. We also found that the DCM+PSS-NP group had markedly higher concentrations of eNOS, NO and VEGFA than other treatment groups. These results showed that PSS-NP could ameliorate the cardiac microvascular endothelial function by promoting the secretion of eNOS, NO and VEGFA $(P<0.05$ or $P<0.01)$.

Furthermore, the levels of PI3K-p85, VEGFA, p-Akt and p-eNOS in DCM group were all significantly lower than those of control group $(P<0.01)$ (Figs.4G-4I). Compared with DCM group, all treatment groups notably increased the protein expression of PI3K-p85 and VEGFA, and the phosphorylation of Akt and eNOS $(\mathrm{P}<0.05$ or $\mathrm{P}<0.01)$. DCM+PSS-NP group was demonstrated to have 
the best effects than other treatment groups $(P<0.05$ or $P<$ 0.01). These results indicated that PSS-NP could improve
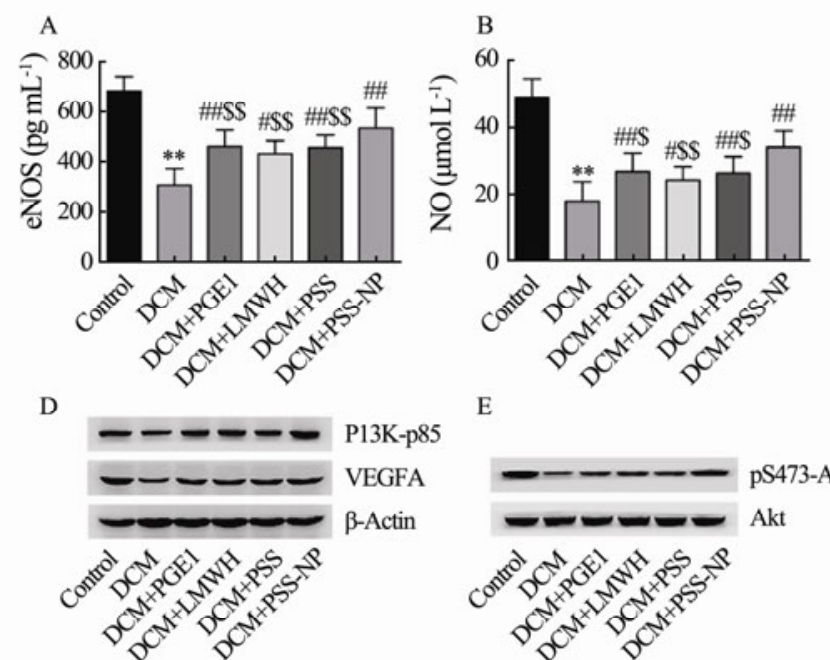

E

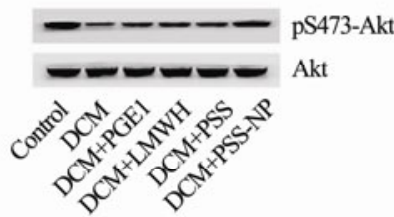

F
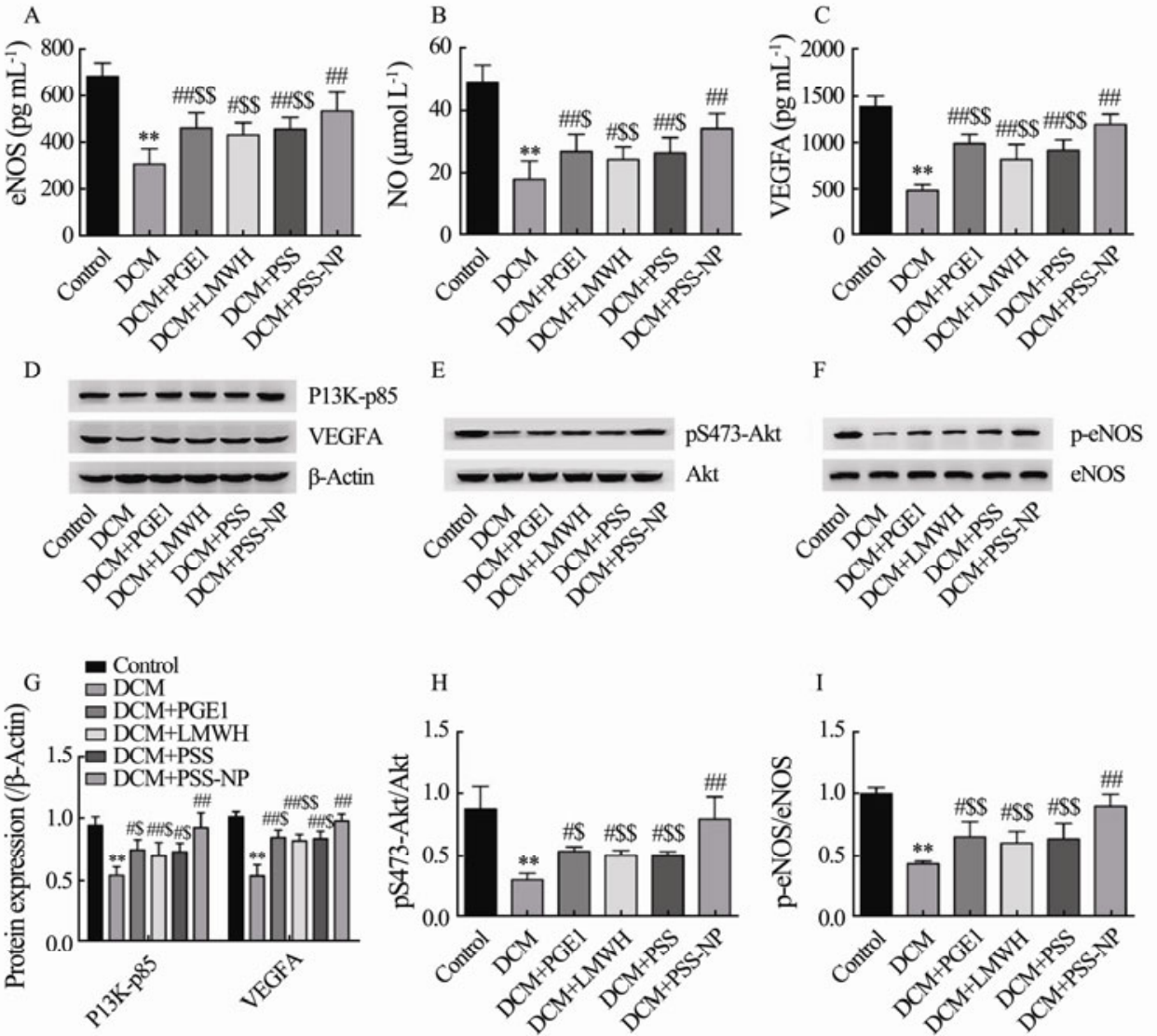

Fig.4 The eNOS, NO, VEGFA and PI3K/Akt/eNOS/VEGFA pathway were detected in all groups. (A-C) The concentration of eNOS and VEGFA in the serum was determined by ELISA kits and NO concentration was detected using NO assay kit. Data are displayed as mean $\pm \mathrm{SD}, \mathrm{n}=6$ per group. (D-F) The PI3K-p85, VEGFA expression and Akt, eNOS phosphorylation levels of left ventricular myocardium tissue were detected by Western Blot in each group (n=6). (G-I) Values were shown in mean $\pm \mathrm{SD}$ from three independent experiments, respectively. ${ }^{*} \mathrm{P}<0.01 \mathrm{vs}$ Control group, ${ }^{\#} \mathrm{P}<0.05$ or ${ }^{\# \#} \mathrm{P}<0.01 \mathrm{vs} \mathrm{DCM}$ group, ${ }^{\$} \mathrm{P}<0.05$ or ${ }^{\$ \$} \mathrm{P}<0.01$ vs DCM+PSS-NP group.

\section{Discussion}

As the incidence of DM rises, DCM causes more and more serious public health problems (Huynh et al., 2014). Recently, clinical studies demonstrated that the cardiac failure in DCM patients might be caused by cardiac microvascular lesion (Yoon et al., 2005). Cardiac microvascular endothelial dysfunction is one of the most important pathophysiological bases of microvascular lesion (Farhangkhoee et al., 2006; Zoungas et al., 2010). It has been proved that PSS plays a significant role in improving microvascular damage, however, with the development of nanotechnology, PSS-NP were prepared by the double (W1/O/W2) emulsion and solvent evaporation methods, used poly lactic-co-glycolic acid (PLGA) as supporter materials. PSS-NP has more outstanding advantages, such as protection of the drug from premature degradation, higher bioavailability, stability and targeting (Zeng et al., 2016; Li et al., 2013; Mizrahy et al., 2012; Elsabahy et al., 2015; Wong et al., 2015).

It has been demonstrated that PSS-NP reduces the fasting blood glucose levels and increases the fasting body weight in type $2 \mathrm{DM}$ rats (Zeng et al., 2016; Li et al., 2013; Zhao et al., 2007). In this study, we investigated the roles of PSS-NP in the type 1 DM rat model, showing that PSS-NP modified the fasting body weight without altering the fasting blood glucose levels.

Consistent with previous findings (Yoon et al., 2005; Wang et al., 2013), our echocardiography results showed that DCM group had overt abnormality of cardiac wall motion and obviously higher LVIDd and LVIDs but lower FS, LVEF and E/A ratio compared with the control group. After 4 weeks of treatment, the results revealed that PSS-NP significantly reversed the progress of cardiac dysfunction in DCM rats.

Previous data demonstrated that cardiac microvascular endothelium was the key target tissue of cardiac microvascular injury in DCM (Kawamura et al., 2017). In this study, the microvascular endothelium ultrastructure in DCM group was changed significantly. The nuclei of endothelial cells were enlarged, the chromatin condensed in margin, and cell membrane showed finger-shaped protuberances to the lumen. We also proved that PSS-NP effectively prevented the progression of such characteristic changes of DCM. In fact, the cardiac microvascular en- 
dothelium morphology in DCM+PSS-NP group were approximately similar to those of the control group.

There are increasing evidences that the PI3K/Akt/ eNOS/VEGFA signaling pathway plays a significant role in cardiac microvascular endothelial function. PI3K is involved in the regulation of cell functions such as proliferation, differentiation and apoptosis. It is shown that under the action of insulin, growth factor and receptor tyrosine kinase (RTK), PI3K can be activated and generate the lipid second messenger phosphatidylinositol-3,4,5trisphosphate (PIP3) on the membrane. PIP3 is combined with Akt's plextrin homology $(\mathrm{PH})$ domain and phosphoinositide-dependent protein kinase(PDK1), which leads to AKT activation through phosphorylating the Thr308 site; however, full activation needs phosphorylate the Ser473 site by rapamycin complex 2 (mTORC2) (Simons et al., 2016; Maillet et al., 2013). As the key effector of PI3K pathway, Akt regulates important cell functions by phosphorylating several downstream factors such as enzymes, kinases, and transcription factors which play critical roles in controlling cell survival, apoptosis, proliferation, growth and angiogenesis (Wu et al., 2009; Chen et al., 2005). It has been revealed that Akt activates the endogenous eNOS-NO system via phosphorylating eNOS. Subsequently, eNOS generates NO in the vascular wall (Alp et al., 2003). NO has some bioactivities including vasodilatation, inhibition of blood cells adhesion to the endothelium, and reduction of vascular endothelial dysfunction caused by free radicals (Forbes et al., 2013; Alp et al., 2003). Some studies have indicated that VEGF is the primary inducer of angiogenesis and the VEGFA isoforms play a central role in vascular development (Xin et al., 2016). The correlation between VEGFA and Akt pathway is complex, as the activation of PI3K-Akt mediates the production of VEGFA, which can induce endothelial cell division, proliferation, migration and angiogenesis. Notably, neovascularization helps to maintain the integrity of the intima (Simons et al., 2016; Wu et al., 2012; Sasso et al., 2005). In this study, we found that PSS-NP-treatment increased serum eNOS, VEGFA and NO levels. We thus speculate that PSS-NP could improve cardiac microvascular endothelial function via promoting secretion of eNOS, NO and VEGFA in DCM rats, but the effects of PSS-NP on cardiac microvascular endothelial function still requires further elucidation. Subsequently, we detected the protein expression of PI3K-p85 and VEGFA, and the phosphorylation of Akt and eNOS by Western blot assay. The protein expression of PI3K-p85 and VEGFA, and the phosphorylation of Akt and eNOS were obviously increased by PSS-NP, which indicated that PSS-NP could improve cardiac microvascular endothelial function via up-regulating the PI3K/Akt/eNOS/VEGFA signaling pathway in STZ-induced DCM rats. Our study provides insight into the role of PSS-NP on cardiac microvascular endothelium in STZ-induced DCM rat model.

In conclusion, we demonstrated an important rule of PSS-NP in improving the structural and functional alterations of cardiac microvascular endothelium. In other words, we inferred that PSS-NP may prevent the dysfunc- tion of cardiac microvascular endothelia in DCM rats by up-regulating the PI3K/Akt/eNOS/VEGFA signaling pathway. These data suggest that PSS-NP may be a novel approach to the treatment the coronary microcirculation disorder diseases such as DCM. PSS-NP is the secondary development of traditional PSS, which is of great significance for the marine biological medicine industry.

\section{Acknowledgements}

This research was supported by grants from the National Natural Science Foundation Project of China (NSFC) (No. 31571829 and No. 31640050) and the People's Livelihood Science and Technology Project Financially Supported by Qingdao city (No. 15-9-2-75-nsh).

\section{References}

Alp, N. J., Mussa, S., Khoo, J., Cai, S., Guzik, T., Jefferson, A., Goh, N., Rockett, K. A., and Channon, K. M., 2003. Tetrahydrobiopterin-dependent preservation of nitric oxide-mediated endothelial function in diabetes by targeted transgenic GTPcyclohydrolase I overexpression. Journal of Clinical Investigation, 112 (5): 725-735, DOI: 10.1172/JCI17786.

Chen, J., Somanath, P. R., Razorenova, O., Chen, W. S., Hay, N., Bornstein, P., and Byzova, T. V., 2005. Akt1 regulates pathological angiogenesis, vascular maturation and permeability in vivo. Nature Medicine, 11 (11): 1188-1196, DOI: 10.1038/ nm1307.

Dong, B., Yu, Q. T., Dai, H. Y., Gao, Y. Y., Zhou, Z. L., Zhang, L., Jiang, H., Gao, F., Li, S. Y., Zhang, Y. H., Bian, H. J., Liu, C. X., Wang, N., Xu, H., Pan, C. M., Song, H. D., Zhang, C., and Zhang, Y., 2012. Angiotensin-converting enzyme-2 overexpression improves left ventricular remodeling and function in a rat model of diabetic cardiomyopathy. Journal of the American College of Cardiology, 59 (8): 739-747, DOI: 10.1016/j.jacc. 2011.09.071.

Elsabahy, M., Heo, G. S., Lim, S. M., Sun, G., and Wooley, K. L., 2015. Polymeric Nanostructures for Imaging and Therapy. Chemical Reviews, 115 (19): 10967-11011, DOI: 10.1021/acs. chemrev.5b00135.

Fang, Z. Y., Prins, J. B., and Marwick, T. H., 2004. Diabetic cardiomyopathy: evidence, mechanisms, and therapeutic implications. Endocrine Reviews, 25 (4): 543-567, DOI: 10.1210 /er.2003-0012.

Farhangkhoee, H., Khan, Z. A., Kaur, H., Xin, X., Chen, S., and Chakrabarti, S., 2006. Vascular endothelial dysfunction in diabetic cardiomyopathy: pathogenesis and potential treatment targets. Pharmacology \& Therapeutics, 111 (2): 384-399, DOI: 10.1016/j.pharmthera.2005.10.008.

Feng, S. S., Mei, L., Anitha, P., Gan, C. W., and Zhou, W., 2009. Poly(lactide)-vitamin E derivative/montmorillonite nanoparticle formulations for the oral delivery of Docetaxel. Biomaterials, 30 (19): 3297-3306, DOI: 10.1016/j.biomaterials. 2009.02.045.

Forbes, J. M., and Cooper, M. E., 2013. Mechanisms of diabetic complications. Physiological Reviews, 93 (1): 137-188, DOI: 10.1152/physrev.00045.2011.

Green, D., Hirsh, J., Heit, J., Prins, M., Davidson, B., and Lensing, A. W., 1994. Low molecular weight heparin: A critical analysis of clinical trials. Physiological Reviews, 46 (1): 89109. 
Hasegawa, H., and Ichioka, S., 2015. Effects of lipo-prostaglandin E1 on wound bed microcirculation. Journal of Wound Care, 24 (7): 293-294, 296, 298-299, DOI: 10.12968/jowc.2015.24. 7.293 .

Hoehn, K. L., Hohnen-Behrens, C., Cederberg, A., Wu, L. E., Turner, N., Yuasa, T., Ebina, Y., and James, D. E., 2008. IRS1-independent defects define major nodes of insulin resistance. Cell Metabolism, 7 (5): 421-433, DOI: 10.1016/j. cmet.2008.04.005.

Huynh, K., Bernardo, B. C., McMullen, J. R., and Ritchie, R. H., 2014. Diabetic cardiomyopathy: Mechanisms and new treatment strategies targeting antioxidant signaling pathways. Pharmacology \& Therapeutics, 142 (3): 375-415, DOI: 10. 1016/j.pharmthera.2014.01.003.

Kawamura, M., Paulsen, M. J., Goldstone, A. B., Shudo, Y., and Woo, Y. J., 2017. Tissue-engineered smooth muscle cell and endothelial progenitor cell bi-level cell sheets prevent progression of cardiac dysfunction, microvascular dysfunction, and interstitial fibrosis in a rodent model of type 1 diabetes-induced cardiomyopathy. Cardiovascular Diabetology, 16 (1): 142, DOI: 10.1186/s12933-017-0625-4.

Li, P. L., Li, C. X., Xue, Y. T., Li, H. H., Liu, H. B., He, X. X., Yu, G. L., and Guan, H. S., 2013. An HPLC method for microanalysis and pharmacokinetics of marine sulfated polysaccharide PSS-loaded poly lactic-co-glycolic acid (PLGA) nanoparticles in rat plasma. Marine Drugs, 11 (4): 1113-1125, DOI: $10.3390 / \mathrm{md} 11041113$.

Litwin, S. E., Raya, T. E., Anderson, P. G., Daugherty, S., and Goldman, S., 1990. Abnormal cardiac function in the streptozotocin-diabetic rat. Changes in active and passive properties of the left ventricle. Journal of Clinical Investigation, 86 (2): 481-488, DOI: 10.1172/JCI114734.

Liu, Y., Lei, S., Gao, X., Mao, X., Wang, T., Wong, G.. T., Vanhoutte, P. M., Irwin, M. G., and Xia, Z., 2012. PKCbeta inhibition with ruboxistaurin reduces oxidative stress and attenuates left ventricular hypertrophy and dysfunction in rats with streptozotocin-induced diabetes. Clinical Science, 122 (4): 161-173, DOI: 10.1042/CS20110176.

Maillet, M., van Berlo, J. H., and Molkentin, J. D., 2013. Molecular basis of physiological heart growth: Fundamental concepts and new players. Nature Reviews Molecular Cell Biology, 14 (1): 38-48, DOI: 10.1038/nrm3495.

Mizrahy, S., and Peer, D., 2012. Polysaccharides as building blocks for nanotherapeutics. Chemical Society Reviews, 41 (7): 2623-2640, DOI: 10.1039/c1cs15239d.

Saito, H., Godo, S., Sato, S., Ito, A., Ikumi, Y., Tanaka, S., Ida, T., Fujii, S., Akaike, T., and Shimokawa, H., 2018. Important role of endothelial caveolin-1 in the protective role of endothelium-dependent hyperpolarization against Nitric Oxide-mediated nitrative stress in microcirculation in mice. Journal of Cardiovascular Pharmacology, 71 (2): 113-126, DOI: 10.1097/FJC.0000000000000552.

Sasso, F. C., Torella, D., Carbonara, O., Ellison, G. M., and Salvatore, T., 2005. Increased vascular endothelial growth factor expression but impaired vascular endothelial growth factor receptor signaling in the myocardium of type 2 diabetic patients with chronic coronary heart disease. Journal of the American College of Cardiology, 46 (5): 827-834, DOI: 10. 1016/j.jacc.2005.06.007.

Simons, M., Gordon, E., and Claesson-Welsh, L., 2016. Mechanisms and regulation of endothelial VEGF receptor signalling. Nature Reviews Molecular Cell Biology, 17 (10): 611-625, DOI: $10.1038 / \mathrm{nrm} .2016 .87$.
Stefek, M., Sotnikova, R., Okruhlicova, L., Volkovova, K., Kucharska, J., Gajdosik, A., Tribulova, N., and Gvozdjakova, A., 2000. Effect of dietary supplementation with the pyridoindole antioxidant stobadine on antioxidant state and ultrastructure of diabetic rat myocardium. Acta Diabetologica, 37 (3): 111-117.

Wang, D., Luo, P., Wang, Y., Li, W., Wang, C., Sun, D., Zhang, R., Su, T., Ma, X., Zeng, C., Wang, H., Ren, J., and Cao, F., 2013. Glucagon-like peptide-1 protects against cardiac microvascular injury in diabetes via a cAMP/PKA/Rho-dependent mechanism. Diabetes, 62 (5): 1697-1708, DOI: 10.2337/db121025.

Wong, P. T., and Choi, S. K., 2015. Mechanisms of drug release in nanotherapeutic delivery systems. Chemical Reviews, 115 (9): 3388-3432, DOI: 10.3390/ijms16011772.

Wu, B., Zhang, Z., Lui, W., Chen, X., Wang, Y., Chamberlain, A. A., Moreno-Rodriguez, R. A., and Zhou, B., 2012. Endocardial cells form the coronary arteries by angiogenesis through myocardial-endocardial VEGF signaling. Cell, 151 (5): 10831096, DOI: 10.1016/j.cell.2012.10.023.

Wu, Y. T., Tan, H. L., Huang, Q., Ong, C. N., and Shen, H. M., 2009. Activation of the PI3K-Akt-mTOR signaling pathway promotes necrotic cell death via suppression of autophagy. Autophagy, 5 (6): 824-834.

Wu, Z., Huang, A., Yan, J., Liu, B., Liu, Q., Zhang, J., Zhang, X., Ou, C., and Chen, M., 2017. Resveratrol ameliorates cardiac dysfunction by inhibiting apoptosis via the PI3K/Akt/FoxO3a pathway in a rat model of diabetic cardiomyopathy. Journal of Cardiovascular Pharmacology, 70 (3): 184-193, DOI: 10. 1097/FJC.0000000000000504.

Xin, H., Zhong, C., Nudleman, E., and Ferrara, N., 2016. Evidence for pro-angiogenic functions of VEGF-Ax. Cell, 167 (1): 275-284, DOI: 10.1016/j.cell.2016.08.054.

Xin, M., Ren, L., Sun, Y., Li, H. H., Guan, H. S., He, X. X., and Li, C. X., 2016. Anticoagulant and antithrombotic activities of low-molecular- weight propylene glycol alginate sodium sulfate (PSS). European Journal of Medicinal Chemistry, 114: 33-40, DOI: 10.1016/j.ejmech.2016.02.063.

Yin, Y., Qi, F., Song, Z., Zhang, B., and Teng, J., 2014. Ferulic acid combined with astragaloside IV protects against vascular endothelial dysfunction in diabetic rats. Bioscience Trends, 8 (4): 217-226.

Zeng, Y., Yang, D., Qiu, P., Han, Z., Zeng, P., He, Y., Guo, Z., Xu, L., Cui, Y., Zhou, Z., Zhang, M., Hao, J., and Zhang, L., 2016. Efficacy of heparinoid pss in treating cardiovascular diseases and beyond-A review of 27 years clinical experiences in China Clinical and Applied Thrombosis/Hemostasis, 22 (3): 222-229, DOI: $10.1177 / 1076029614551822$.

Zhang, X., Pan, L., Yang, K., Fu, Y., Liu, Y., Chi, J., Zhang, X., Hong, S., Ma, X., and Yin, X., 2017. H3 relaxin protects against myocardial injury in experimental diabetic cardiomyopathy by inhibiting myocardial apoptosis, fibrosis and inflammation. Cellular Physiology and Biochemistry, 43 (4): 1311-1324, DOI: 10.1159/000481843.

Zhao, M. M., Li, Z., Teng, Z., Zhao, J. S., Yu, X. H., Watanabe, Y., and Zhao, L. M., 2007. Repeated oral treatment with polysaccharide sulfate reduces insulin resistance and dyslipidemia in diabetic dyslipidemic rat model. Acta Pharmaceutica Sinica, 42 (5): 488-491.

Zoungas, S., Patel, A., Chalmers, J., de Galan, B. E., Li, Q., Billot L., Woodward, M., Ninomiya, T., Neal, and Group, A. C., 2010. Severe hypoglycemia and risks of vascular events and death. New England Journal of Medicine, 363 (15): 1410-1418, DOI: 10.1056/NEJMoa1003795.

(Edited by Ji Dechun) 